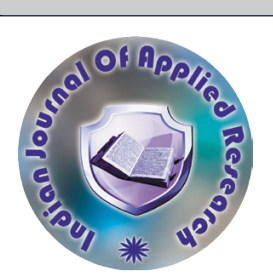

Cardiology

\title{
COMPARISON OF CONDUIT PATENCY AND ROLE OF \\ ANTICOAGULANTS IN LONGITIVTIY OF PROSTHETIC GRAFT VERSUS AUTOLOGOUS SAPHENOUS VEIN FOR BELOW KNEE BYPASS GRAFTING IN CRITICAL LIMB ISCHEMIA
}

Dr. Nishant Singh Chandel

Dr. Krishnakant Sahu*
(m.Ch.) Department Of Cardiovascular And Thoracic Surgery Pt. J.N.M. Medical College And B.R.A.M Hospital ,Raipur (C.G.)

(m.Ch.) Department Of Cardiovascular And Thoracic Surgery Pt. J.N.M. Medical College And B.R.A.M Hospital ,Raipur (C.G.) *Corresponding Author

\section{ABSTRACT}

BACKGROUND: As established fact the autogenous vein is the conduit of choice in below-knee arterial bypasses However, with availability of newer prosthetic grafts and usage of anti-platelets and anticoagulants , the results of these prosthesis's are also improving. so much so that in case of non availability of good GSV or malnourished patients where wound healing could be a concern prosthetic grafts can be used reasonably well.

We also compared the outcomes of below-knee prosthetic versus autologous vein bypass grafts with different anti-platelets and anti-thrombotic medicines combinations to find out whether the prosthesis performance improves with anticoagulants usage and whether the addition of anticoagulants to anti-platelets is causing more bleeding complications in these patients so much that they are to be used with caution.

METHODS: For this study, we utilised treatment data of Department of CardioVascular and Thoracic Surgery ,Pt. J.N.M. Medical College Raipur. we studied 70 patients, Single surgeon experience. We included patients who underwent open below knee bypass procedure for critical limb ischemia (claudication/ rest pain/ non healing ulcers/ gangrenous changes) between January 1, 2018 and December 31, 2019. Our analysis was limited to patients whose graft origins were the ipsilateral iliac or femoral arteries and whose targets were the below-knee popliteal or tibial arteries.

We analysed the results by evaluating the Graft Patency at 1 year, major amputation at 1 year, bleeding complications, association of bleeding complications to the combination of anti-platelets + anticoagulation.

we also evaluated the patency at 1 year in relation to the distal anastomotic sites ( popliteal or infra popliteal ) and amputation in relation to the distal targets for bypass to understand that the disease load has any significance in the patency and limb salvage in patients of lower limb ischemia.

RESULTS :A total of 70 patients who underwent open below knee bypass procedure for critical limb ischemia were analysed; 35 patients (50\%) received GSV and 35 patients $(50 \%)$ received a prosthetic conduit. There was no significant difference in primary patency due to Gender (Male 84.3\%, Female 15.7\%), Diabetes Mellitus (GSV 20\%, Prosthetic 14.3\%), Hypertension (GSV 31.4\%, Prosthetic 45.7\%) \& Tobacco addition (GSV $100 \%$, Prosthetic 94.3\%). Baseline characteristics were similar among groups with the popliteal artery (54.3\%) and infra popliteal arteries. i.e. tibioperoneal trunk (27.1\%), Anterior Tibial (4.3\%) and posterior tibial (14.3\%). We found no significant difference in primary graft patency $(77 \%$ vs $71 \%, \mathrm{P}=)$ or major amputation rates $(8.5 \%$ vs $17 \%, \mathrm{P}=)$ between GSV and Prosthetic conduit. The prosthetic graft patency was more when the anti platelets were combined with anticoagulants. Saphenous vein graft patients did well even with anti platelets single or combination. Bleeding complications were more common in the prosthetic group with anti platelets with anticoagulants.

CONCLUSIONS:Although limited in size, our study demonstrates that, with appropriate patient selection and anti-thrombotic therapy, 1 -year outcomes for below-knee prosthetic bypass graft can be comparable to those for greater saphenous vein conduit.Though the study does not challenge the superiority of vein graft for below knee bypass grafting, but in certain cases where needed prosthetic graft are definitely comparable in performance.

\section{KEYWORDS : Anti-platelets, Anti-thrombotic, Prosthetic Grafts, Gsv Grafts}

\section{INTRODUCTION}

Type of conduit and the site of below knee distal arterial anastomosis for popliteal and infra popliteal arteries are relevant predictors of infrainguinal bypass patency. Autogenous reversed GSV maintains superior patency compared with prosthetic grafts (e-PTFE or Dacron grafts). Short- segment prosthetic bypasses to above-knee targets can approach the approximately $80 \% 5$-year patency rates as obtained with saphenous vein grafts. But longer prosthetic grafts crossing the joint usually do not perform as well as seen in several retrospective series.

However, many patients with long-segment occlusions lack adequate great saphenous vein, either due to inadequate size , superficial thrombophlebitis or because it has been previously harvested. Adjunctive use of anti-platelets with or without anti-thrombotic therapy has been proposed to improve outcomes. Single anti-platelet therapy (Ecosprin) was associated with a approximately $40 \%$ relative risk reduction of venous graft occlusion. For prosthetic grafts that crosses the knee joint, a recently published randomised trial found that the addition of clopidogrel to aspirin contributed an additional $35 \%$ to $40 \%$ reduction of graft thrombosis. Anticoagulation with warfarin or acitrom may provide a protective effect for below knee prosthetic bypass grafts, especially to infra popliteal targets.

\section{METHODS:}

\section{DATABASE AND SUBJECTS}

For this study, we utilised data from Department of CardioVascular and Thoracic Surgery ,Pt. J.N.M. Medical College Raipur. we studied 70 patients, single surgeon experience. Patient-level and operative data were procured Pre Operative, Peri Operative period and at 1-year follow-up.
We included patients who underwent open below knee bypass procedure for critical limb ischemia (claudication/ rest pain/ non healing ulcers/ gangrenous changes) between January 1, 2018 and December 31,2018. Our analysis was limited to patients whose graft origins were the ipsilateral iliac or femoral arteries and whose targets were the below-knee popliteal or tibial arteries.

Lower extremity bypass grafts that did not cross the knee joint were excluded. To allow comparison of prosthetic conduit to an "ideal" conduit, we studied only patients who underwent surgery with either a single-segment great saphenous vein or prosthetic $25(71.4 \%)$ epolytetrafluoroethylene and 10 (28.6\%) Dacron). We also excluded patients who lacked sufficient follow-up data (4\%). Mean follow-up time for the cohort was 400 days.

\section{DEFINITIONS OF ANTI-THROMBOTIC USE}

Patients were kept on anti-platelet (single or dual i.e. ecosprin +/clopidogrel) depending upon tolerance of patients, for vein grafts. where as the prosthetic graft patients were kept on anticoagulation with or without anti-platelets. The anti-thrombotic medication utilised in our study was universal acitrom (nicoumalone). Patients were recorded to be taking these medications preoperatively (ecosprin till the date of surgery, clopidogrel and acitrom stopped 3 days prior to surgery), at hospital discharge after surgery, and till completion of 1year follow-up.

We studied patients with a venous graft vs prosthetic graft, who were on aspirin alone $(n=23)$, aspirin plus clopidogrel $(n=19)$, aspirin plus acitrom $(n=22)$, and aspirin plus clopidogrel plus acitrom $(n=6)$. Comparisons between outcomes were performed across these diffe 
Volume -10 | Issue - 3 | March - 2020 | PRINT ISSN No. 2249 - 555X | DOI : 10.36106/ijar

Table No. 2: Distribution of Operative characteristics of the two

\section{MATCHING COHORTS}

Decisions regarding conduit type and anti-thrombotic treatment were made by the treating Vascular surgeon. To control for the nonrandom decision to utilise an autologous or prosthetic bypass conduit, we used propensity matching methods to create similar patient cohorts. We matched patients who received a prosthetic conduit to patients with a greater saphenous vein conduit by stratified propensity score . This ensured that our two cohorts were matched equally in terms of age, gender, comorbidity, anticoagulation therapy, indication, and operative details.

\section{DEFINITIONS OF OUTCOME MEASURES}

Our main outcome measures were primary graft patency and the incidence of major lower extremity amputation. Primary patency was defined as uninterrupted patency of the bypass graft with no requirement of procedure or intervention of the conduit itself after implantation for 1 year duration of followup. Patency was assessed by physical examination and duplex scan, sometimes CT Angiogram.

Below-knee and above-knee amputations qualified as major leg amputations due to extension of gangrene after surgery. Some cases came with established gangrene but post surgery the gangrene did not extend and thus the level of amputation was lowest possible and thus they were not considered as major amputation.

Secondary outcomes assessed were patient survival and bleeding complications. We defined a bleeding complication as bleeding (overt or occult) requiring blood transfusion of $>2$ units of packed red blood cells.

\section{STATISTICALANALYSIS}

When comparing patient demographics, we applied chi-squared analysis, proportion test for categorical variables, depending on the number of groups compared. The chi-squared analysis was used to compare bleeding complication, Primary patency at 1 years follow up \& Major Amputation. The 95\% confidence intervals (CIs) were reported when appropriate and $\mathrm{P}<0.05$ was considered as a Significant

All analyses were performed using Microsoft Excel \& SPSS 20.0 version Software.

\section{RESULTS}

We studied 70 patients who undergone below knee bypass surgery for critical limb ischemia between Jan. 2018 and Dec. 2019 and met our inclusion criteria. Demographic were similar between the two groups for Age, Sex and there is no statistically significant differences between the two groups. Morbidity wise diabetes was found in 12 (17.1\%), Hypertension in $27(38.6 \%)$ (Table 1$)$. Whereas coronary artery disease was found in $3(4.3 \%)$ The incidence of diabetes mellitus was higher in the GSV group but this did not reach statistical significance.

Table No. 1: Patients demographic of the two groups.

\begin{tabular}{|c|c|c|c|}
\hline Characteristics & GSV & Prosthetic & P Value \\
\hline $\operatorname{Sex}(\mathrm{M} / \mathrm{F})$ & $29 / 6$ & $30 / 5$ & 0.743 \\
\hline \multicolumn{4}{|l|}{ Age } \\
\hline $40-49$ & $11(31.4 \%)$ & $19(54.3 \%)$ & \multirow[t]{4}{*}{0.181} \\
\hline $50-59$ & $13(37.1 \%)$ & $11(31.4 \%)$ & \\
\hline $60-69$ & $10(28.6 \%)$ & $4(1.4 \%)$ & \\
\hline $70-79$ & $1(2.8 \%)$ & $1(2.8 \%)$ & \\
\hline Tobacco Addiction & $35(100.0 \%)$ & $33(94.3 \%)$ & 0.153 \\
\hline Diabetes Mellitus & $7(20.0 \%)$ & $5(14.3 \%)$ & 0.529 \\
\hline Hypertension & $11(31.4 \%)$ & $16(45.7 \%)$ & 0.219 \\
\hline CAD & $2(5.7 \%)$ & $1(2.8 \%)$ & 0.555 \\
\hline
\end{tabular}

The baseline characteristics and comparisons of the two groups are summarised in table 2. Anti platelets and anti thrombotic procedures were less frequent in the GSV group compared with the Prosthetic group. The prosthetic group had a significantly higher rate of popliteal targets and more frequent use of anticoagulation. Ischemic rest pain was noted in $56(80 \%)$ and tissue loss in $39(55.7 \%)$ patients. Of these, $35(50 \%)$ received greater saphenous vein (GSV) and $35(50 \%)$ received prosthetic conduit.

\section{groups.}

\begin{tabular}{|c|c|c|c|}
\hline Characteristics & GSV & Prosthetic & P Value \\
\hline \multicolumn{4}{|l|}{ Graft Origin } \\
\hline External Iliac & $6(17.1 \%)$ & $9(25.7 \%)$ & \multirow[t]{3}{*}{0.535} \\
\hline Common Femoral & $14(40 \%)$ & $15(42.9 \%)$ & \\
\hline Superficial Femoral & $15(42.9 \%)$ & $11(31.4 \%)$ & \\
\hline \multicolumn{4}{|l|}{ Graft Recipient } \\
\hline Below Knee Popliteal & $18(51.4 \%)$ & $20(57.1 \%)$ & \multirow[t]{4}{*}{0.473} \\
\hline Tibioperoneal trunk & $8(22.9 \%)$ & $11(31.4 \%)$ & \\
\hline Ant. Tibial & $2(5.7 \%)$ & $1(2.9 \%)$ & \\
\hline Post Tibial & $7(20 \%)$ & $3(8.6 \%)$ & \\
\hline \multicolumn{4}{|c|}{ Anti Platelets / Anti thrombotic } \\
\hline Aspirin & $20(57.1 \%)$ & $3(8.6 \%)$ & \multirow[t]{4}{*}{$<0.001$} \\
\hline Aspirin + Clopidogrel & $11(31.4 \%)$ & $8(22.9 \%)$ & \\
\hline Aspirin + Acitrom & $4(11.4 \%)$ & $18(51.4 \%)$ & \\
\hline $\begin{array}{l}\text { Aspirin + Clopidogrel } \\
+ \text { Acitrom }\end{array}$ & $0(0.0 \%)$ & $6(17.1 \%)$ & \\
\hline
\end{tabular}

A comparison of outcomes between the group of patients receiving GSV and the group of patients receiving a prosthetic conduit is shown in table 3. Bleeding complications were more in anti-platelet + anticoagulation group . out of 70 patients , $42(60 \%)$ patients received a anti-platelets (single or dual) and $28(40 \%)$ patients received antiplatelets + anticoagulants. out of anti-platelets group only 3 patients $(7 \%)$ had significant bleeding complications. whereas 14 patients $(32 \%)$ in anti-platelets + anticoagulant group had bleeding complications. Primary graft patency at 1 year was $77 \%$ of GSV and $71.4 \%$ of Prosthetic group $(\mathrm{P}=0.979)$. Patients with a prosthetic graft were more often treated with acitrom in addition to aspirin $(51.4 \%$ vs. $11.4 \%$ or with acitrom in addition to aspirin and clopidogrel $(17 \%$ vs. $0 \%$ ). Conversely, patients with GSV were more commonly treated with aspirin alone $57 \%$ vs. $8.5 \%$ ) or with aspirin plus clopidogrel $(31.4 \%$ vs. $22.8 \%)$. Major limb amputation occurred in $8.5 \%$ of patients with GSV and $17 \%$ of patients with a prosthetic graft

Table No.3: Comparison of outcomes between GSV \& Prosthetic grafts using Anti thrombotic therapy.

\begin{tabular}{|l|l|l|l|}
\hline Anti Thrombotic Therapy & GSV & Prosthetic & P Value \\
\hline Bleeding Complications & 0 & 0 & 0.999 \\
\hline Aspirin & 1 & 2 & 0.953 \\
\hline Aspirin + Clopidogrel & 3 & 4 & 0.979 \\
\hline Aspirin + Acitrom & 0 & 7 & 0.793 \\
\hline Aspirin + Clopidogrel + Acitrom & 0 & 13 & 0.825 \\
\hline Total & 4 & \multicolumn{5}{l|}{} \\
\hline Primary Patency at 1 Year & 13 & 0 & 0.721 \\
\hline Aspirin & 10 & 6 & 0.917 \\
\hline Aspirin + Clopidogrel & 4 & 14 & 0.811 \\
\hline Aspirin + Acitrom & 0 & 5 & 0.826 \\
\hline Aspirin + Clopidogrel + Acitrom & 0 & 25 & 0.979 \\
\hline Total & 27 & \multicolumn{5}{|l|}{} \\
\hline Major Amputation at 1 Year & 2 & 3 & 0.964 \\
\hline Aspirin & 0 & 2 & 0.886 \\
\hline Aspirin + Clopidogrel & 1 & 1 & 0.999 \\
\hline Aspirin + Acitrom & 0 & 1 & 0.919 \\
\hline Aspirin + Clopidogrel + Acitrom & 0 & 7 & 0.923 \\
\hline Total & 3 &
\end{tabular}

Primary outcomes analysed based on whether patients had their distal anastomosis at the below-knee popliteal artery (or tibioperoneal trunk) vs at a tibial vessel. Those who had a venous conduit to the below-knee popliteal artery maintained $80 \%$ primary graft patency and those grafted at infra popliteal level had $66.7 \%$ patency at 1 year . Patients with a prosthetic graft to the same target level had a primary patency rate of $77.4 \%$ at popliteal level and $25 \%$ at infra popliteal arteries. The incidence of amputation in patients with venous grafts targeting the popliteal arteries was $7.7 \%$ and in prosthetic group was $16 \%$. whereas $\mathrm{t}$ in infra popliteal targets amputation rate was $22.6 \%$ in GSV, and $50 \%$ in prosthetic group.

we could easily see that the bleeding complications were more oftenly seen in dual anti-platelet + anticoagulation group (35\%) and single anti-platelet + anti coagulation group (35\%) than only anti-platelets 
group (15\%). this did not have any association to the type of graft, though the prothetic graft patients were kept on higher antiplatelet+anticoagulation medicines, so this appeared that bleeding is more in prosthetic than GSV group.

we noted a higher amputation rate in the subgroup of patients who were only on aspirin and received a prosthetic versus venous conduit .

\section{DISCUSSION}

Since the autogenous saphenous vein was first described as a bypass conduit for femoral arterial disease in 1949, it has remained the conduit of choice. However, many patients with tissue loss and superficial thrombophlebitis due to various reasons autologous saphenous vein remains unavailable for quarter of bypass patients.

These patients often receive a prosthetic bypass conduit as now there are better and improved bypass graft conduits are available, they can be second option to autologous vein but are definitely satisfactory option, especially in the era of adjunctive anti-platelets and anticoagulants.

In our study, we compared 1-year outcomes of below knee bypass for patients with critical limb ischemia who received a prosthetic conduit with those who received time tested reversed great saphenous vein. Surprisingly, we discovered little difference in primary graft patency, and amputation rates but more bleeding complications within the first year of surgery and only marginal clinical and statistical benefit in terms of limb salvage and survival for those patients who received a saphenous vein bypass

\section{WE BELIEVE THE POSSIBLE EXPLANATIONS COULD BE}

1. Too early to note a difference. Our initiative evaluated patients at 1-year follow- up, which limits our ability to assess long-term outcomes in graft patency and limb salvage. It his highly likely that, in long term the venous grafts outperform the prosthetic grafts but after 1 year, patency rates for prosthetic conduit was almost equally good

Prosthetic graft patients were more commonly on anti-coagulations + anti-platelets .mostly this combination of medicines were responsible for comparable patency of the prosthesis group, resulting in the lack of difference in outcomes by conduit type.

For example, the CASPAR trial demonstrated that, in a controlled environment, the addition of a second anti-platelet agent (clopidogrel plus aspirin) provides a greater protective effect for infra geniculate prosthetic bypass grafts when compared with aspirin alone. so this finding led us use higher doses of anti-platelts and anti-coagulations, so that the coagulability of blood was less and so the graft thrombosis.

Brumberg and colleagues reported that therapeutic warfarin use in low-flow below-knee prosthetic grafts is associated with significantly improved patency rates. Further, a single centre randomisation of patients with infrainguinal prosthetic bypass grafts mirrored these findings. Sarac and colleagues detailed an improvement in 3-year primary graft patency by $50 \%$ in patients who received warfarin in addition to base-fine aspirin.

However, although some studies supported the use of warfarin to preserve graft patency, others have refuted this assumption. A large multi-centre study in Europe (Dutch Bypass Oral Anticoagulants or Aspirin Study) demonstrated that oral anticoagulants were not associated with improved graft patency for prosthetic femoropopliteal or femorocrural grafts. Further, in a study from UCLA, a 20-year review of infrageniculate bypass surgery with prosthetic conduit showed no association between warfarin use and prosthetic bypass patency.

But in our study we used anticoagulants in view that our region blood hyper-coagulability is common observation, patients are non comp liant in abstinence from tobacco, dehydration is common in labourer class, hyper-homocystienemia is endemic in this region.

Nevertheless, study would require stronger evidence than our observational data owing to small sample size and short term followup , could provide. Future work addressing the interaction between conduit types and adjunctive anti-thrombotic regimens, in a controlled setting, will be needed to reach such conclusions. we propose that the patients with this problem can be evaluated with detailed coagulation profile, if the blood characteristics are to wards procoagulant state, adding the anticoagulants will be more justified.

Our study has other limitations. we noticed that the group of patients who were having poor veins and received prosthetic grafts, their atherosclerotic burden was high and had poor runoff vessels. This could have negative effect on the longitivity of the conduit. Moreover we did not practice the vein cuff for below knee tibial level prosthetic grafts. We seriously think that when the distal target is any of the tibial arteries , adding a small piece of vein between the prosthetic graft and tibial artery can improve the results of prosthetic graft to tibial level grafting.

Our analysis of bleeding complications was limited to short-term occurrences. We cannot comment on long-term adverse effects that are often associated with chronic anticoagulation, such as hematomas or cerebral bleeds, as our registry does not capture these. Our observation that prosthetic graft group had more bleeding, owing to anti-platelets + anti-coagulations combinations, our major deterrent was poor timely followup of patients in PT/INR testing leading to occasional bleeding incidence due to fluctuating INR values causing bleeding issues. Those with acceptable INR values and regular followups were less likely to have bleeding incidences.

In conclusion, our investigation has shown that, within a 1-year period, prosthetic below knee bypass grafts can be equally good conduit as saphenous vein grafts given appropriate patient selection and antithrombotic therapy.Further controlled trials, especially those investigating novel anti-thrombotic therapies, are necessary to better delineate the use of protective adjuncts with prosthetic bypass conduits. Nonetheless, given the statistically different limb salvage rates, single-segment GSV, when available, remains the preferred conduit.

\section{REFERENCES}

1. Comparison of Graft Patency, Limb Salvage, and Anti-thrombotic Therapy Between Prosthetic and Autogenous Below-Knee Bypass for Critical Limb Ischemia Bjoern D. Suckow, Larry W. Kraiss, David H. Stone, Andres Schanzer, Ann Vasc Surg. Author manuscript; available in PMC 2014 December

2. Archie JP Jr. Femoropopliteal bypass with either adequate ipsilateral reversed saphenous vein or obligatory polytetrafluoroethylene. Ann Vasc Surg. 1994; 8:475-84. [PubMed: 7811585]

3. Nicoloff AD, Taylor LM Jr, McLafferty RB, Moneta GL, Porter JM. Patient recovery after infrainguinal bypass grafting for limb salvage. J Vasc Surg. 1998; 27:256-63. [PubMed: 9510280]

4. Green RM, Abbott WM, Matsumoto T, et al. Prosthetic above knee femoropopliteal bypass grafting: five-year results of a randomized trial. J Vasc Surg. 2000; 31:417-25. [PubMed: 10709052

5. Abu Rahma AF, Robinson PA, Holt SM. Prospective controlled study of polytetrafluoroethylene versus saphenous vein in claudicant patients with bilateral above knee femoropopliteal bypasses. Surgery. 1999; 126:594-601. [PubMed: above knee 10520903 ]

6. Schweiger H, Klein P, Lang W. Tibial bypass grafting for limb salvage with ringed polytetrafluoroethylene prostheses: results of primary and secondary procedures. J Vasc Surg. 1993; 18:867-74. [PubMed: 8230575]

7. Albers M, Battistella VM, Romiti M, Rodrigues AA, Pereira CA. Meta-analysis of polytetrafluoroethylene bypass grafts to infrapopliteal arteries. J Vasc Surg. 2003; 37:1263-9. [PubMed: 12764274]

8. Dorffler-Melly J, Buller HR, Koopman MM, Prins MH. Antithrombotic agents for preventing thrombosis after infrainguinal arterial bypass surgery. Cochrane Database Syst Rev. 2003 Cd000536.

9. Sarac TP, Huber TS, Back MR, et al. Warfarin improves the outcome of infrainguinal vein bypass grafting at high risk for failure. J Vasc Surg. 1998; 28:446-57. [PubMed: 9737454]

10. Brumberg RS, Back MR, Armstrong PA, et al. The relative importance of graf surveillance and warfarin therapy in infrainguinal prosthetic bypass failure. J Vasc Surg. 2007; 46:1160-6. [PubMed: 17920227]

11. Faries PL, Logerfo FW, Arora S, et al. A comparative study of alternative conduits for lower extremity revascularization: all-autogenous conduit versus prosthetic grafts. J Vasc Surg. 2000; 32:1080-90. [PubMed: 11107079]

12. Taylor LM Jr, Edwards JM, Porter JM. Present status of reversed vein bypass grafting. five-year results of a modern series. J Vasc Surg. 1990; 11:193-205. [PubMed: 2299743]

13. Efficacy of oral anticoagulants compared with aspirin after infrainguinal bypass surgery (The Dutch Bypass Oral Anticoagulants or Aspirin Study): a randomised trial. Lancet. 2000;355:346-51. [PubMed: 10665553]

14. Kashyap VS, Ahn SS, Quinones-Baldrich WJ, et al. Infrapopliteal-lower extremity revascularization with prosthetic conduit: a 20 -year experience. Vasc Endovasc Surg. $2002 ; 36: 255-62$

15. Moawad J, Gagne P. Adjuncts to improve patency of infrainguinal prosthetic bypass grafts. Vasc Endovasc Surg. 2003; 37:381-6.

16. Oderich GS, Panneton JM, Yagubyan M, et al. Comparison of precuffed and vein-cuffed expanded polytetrafluoroethylene grafts for infragenicular arterial reconstructions: a
case-matched study. Ann Vasc Surg. 2005; 19:49-55. [PubMed: 15714367]

17. Pappas PJ, Hobson RW 2nd, Meyers MG, et al. Patency of infrainguinal polytetrafluoroethylene bypass grafts with distal interposition vein cuffs. Cardiovasc Surg. 1998; 6:19-26. [PubMed: 9546843]

18. Rowe VL, Kumar SR, Glass H, Hood DB, Weaver FA. Race independently impacts outcome of infrapopliteal bypass for symptomatic arterial insufficiency. Vasc Endovasc Surg. 2007; 41:397-401. 